\title{
On Simulating Subjective Evaluation Using Combined Objective Metrics for Validation of 3D Tumor Segmentation
}

\author{
Xiang Deng ${ }^{1}$, Lei Zhu ${ }^{1}$, Yiyong Sun ${ }^{2}$, Chenyang Xu ${ }^{2}$, Lan Song ${ }^{5}$, \\ Jiuhong Chen ${ }^{3}$, Reto D. Merges ${ }^{3}$, Marie-Pierre Jolly ${ }^{2}$, Michael Suehling ${ }^{4}$, \\ and Xiaodong $\mathrm{Xu}^{1}$ \\ ${ }^{1}$ Corporate Technology, Siemens Ltd., China \\ xiang.deng@siemens.com \\ ${ }^{2}$ Siemens Corporate Research, USA \\ ${ }^{3}$ Medical Solutions, Siemens Ltd., China \\ ${ }^{4}$ Siemens Medical Solutions, Germany \\ ${ }^{5}$ Peking Union Medical College Hospital, China*
}

\begin{abstract}
In this paper, we present a new segmentation evaluation method that can simulate radiologist's subjective assessment of $3 \mathrm{D}$ tumor segmentation in CT images. The method uses a new metric defined as a linear combination of a set of commonly used objective metrics. The weighing parameters of the linear combination are determined by maximizing the rank correlation between radiologist's subjective rating and objective measurements. Experimental results on 93 lesions demonstrate that the new composite metric shows better performance in segmentation evaluation than each individual objective metric. Also, segmentation rating using the composite metric compares well with radiologist's subjective evaluation. Our method has the potential to facilitate the development of new tumor segmentation algorithms and assist large scale segmentation evaluation studies.
\end{abstract}

\section{Introduction}

Tumor volume measured from $\mathrm{CT}$ images has been shown to provide more accurate estimate of lesion size than 1D and 2D measurements 112 in cancer diagnosis and evaluation of treatment response [3]. Quantitative validation of image segmentation techniques for computing lesion volume is essential for routine clinical use of tumor volumetry.

The quality of the lesion segmentation is traditionally evaluated using either subjective or objective methods [4. While the subjective evaluation by radiologists, such as five-level rating [ [5, is considered as gold standard in clinical

\footnotetext{
* We would like to thank Guangwei Du and Haibin Huang at Corporate Technology, Siemens Ltd., China, Dr. Zhengyu Jin at Peking Union Medical College Hospital, China, and Daniel Rinck at Siemens Medical Solutions, Germany for their support on this work.
} 


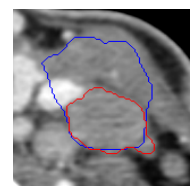

(a)

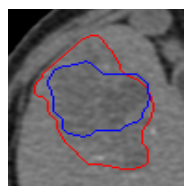

(b)

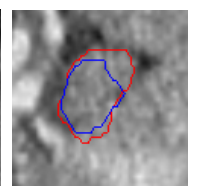

(c)

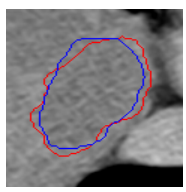

(d)

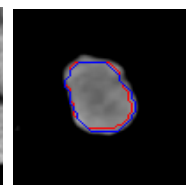

(e)

Fig. 1. Tumor segmentations with scores 1 to 5 ((a)-(e)) rated by a radiologist. The red contours are the ground truth defined by the radiologist. The blue contours are the results from the semi-automatic segmentation technique.

setting, it is costly and time consuming, especially for large scale validation studies. Also, the intra-rater and inter-rater variability might result in inconsistent ratings. Compared to the subjective evaluation, the objective metrics are easy to compute, but may not provide an overall assessment of the segmentation quality because each metric only captures certain aspect of the difference between the segmentation and the ground truth. Recently, several methods based on combined objective metrics have been proposed to provide evaluation more relevant to subjective assessment of generic object segmentation [67/89]. The weights in these combined measures, however, are determined rather empirically, and may not be applicable for tumor segmentation evaluation due to the medical expertise required in the assessment. To date, little work has been done to study radiologist's qualitative evaluation of 3D tumor segmentation.

In this paper, we present a new method for evaluating $3 \mathrm{D}$ tumor segmentation. By quantitatively studying the relation between the subjective and objective measures, we propose a new composite metric that can simulate radiologist's subjective rating. The composite metric is defined as a linear combination of a set of objective metrics. Compared to the previously published empirically defined combined metrics, the weighing parameters of the linear combination in our method are determined by maximizing the Spearman rank correlation coefficient between the composite metric and radiologist's subjective rating. Five cluster centers defined by the composite metric are derived from training data to simulate the five-level scale. A segmentation is rated using the corresponding composite metric value according to the distance to the five cluster centers.

The composite metric in our method shows better performance in rating segmentation than each individual objective metric, and compare well with radiologist's subjective rating. Our method has the potential to facilitate the development of new tumor segmentation algorithms and assist large scale segmentation evaluation studies when radiologist's rating is not available.

\section{Method}

\subsection{Subjective Evaluation and Objective Metrics}

The radiologist's subjective evaluation for the 3D tumor segmentation was obtained in three steps. First, the ground truth for all lesions was defined by an 
experienced radiologist on the CT images. Second, all lesions were segmented using an in-house developed technique based on algorithm described in [10]. Third, the quality of the 3D tumor segmentation was evaluated by the same radiologist using a five-point scale, as shown in Fig. 1, where $1=$ segmentation is highly inaccurate, $2=$ segmentation is moderately inaccurate, $3=$ segmentation is marginally accurate, $4=$ segmentation is moderately accurate, and $5=$ segmentation is highly accurate.

We investigated a total of ten commonly used volume related objective metrics 1 . These metrics reflect different aspects of discrepancy in segmented and ground truth volumes, such as relative position and volume difference. Three of the ten metrics with highest correlation with radiologist's subjective rating were selected as candidates to construct a composite metric, which is described in the next section. The three objective metrics are defined as follows

a) Volume overlap $\left(m_{1}\right)$

$$
m_{1}=\left(\mathrm{Vol}_{\mathrm{seg}} \cap \mathrm{Vol}_{\mathrm{gt}}\right) /\left(\mathrm{Vol}_{\mathrm{seg}} \cup \mathrm{Vol}_{\mathrm{gt}}\right)
$$

where $\mathrm{Vol}_{\text {seg }}$ denotes segmented volume. Vol gt denotes ground truth volume.

b) Absolute value of normalized volume difference $\left(m_{2}\right)$

$$
m_{2}=\left|\mathrm{Vol}_{\mathrm{seg}}-\mathrm{Vol}_{\mathrm{gt}}\right| / \mathrm{Vol}_{\mathrm{gt}}
$$

c) RMS surface distance $\left(m_{3}\right)$

$$
m_{3}=\sqrt{\frac{\sum_{a \in A}\left[\min _{b \in B}\{\operatorname{dist}(\mathrm{a}, \mathrm{b})\}\right]^{2}+\sum_{b \in B}\left[\min _{a \in A}\{\operatorname{dist}(\mathrm{a}, \mathrm{b})\}\right]^{2}}{N_{A}+N_{B}}}
$$

where $A$ and $B$ denote the surfaces of segmented and ground truth volumes. $a$ and $b$ are mesh point on $A$ and $B$ respectively. dist(a, b) denotes the distance between $a$ and $b . N_{A}$ and $N_{B}$ are the number of points on $A$ and $B$.

\subsection{New Composite Metric}

Motivated by the work in [11, we propose a new metric that can simulate the radiologist's subjective rating in segmentation evaluation. It is defined as a linear combination of a set of objective metrics described in previous section,

$$
u=\sum_{i=1}^{n} c_{i} \cdot m_{i}
$$

where $u$ is the composite metric, $c_{i}$ is the weighing parameter, $m_{i}$ is the objective metric, $n$ is the number of objective metrics.

\footnotetext{
${ }^{1}$ The ten volume related objective metrics are volume overlap, over-segmentation ratio, under-segmentation ratio, volume difference, absolute value of volume difference, normalized volume difference, absolute value of normalized volume difference, RMS surface distance, maximum of surface distance, and 75 percentile of surface distance.
} 
Let $Q$ denote the number of lesions used in this study, we can stack the objective measurements obtained from segmentation of all these lesions into the matrix equation

$$
\mathbf{u}=\mathbf{M c} \text {. }
$$

where $\mathbf{u}$ is a $Q \times 1$ vector of the measurements from the composite metric. $\mathbf{M}$ is a $Q \times n$ matrix of the measurements from the objective metrics. c is a $n \times 1$ vector of weighing parameters.

To simulate the radiologist's five-level ratings of 3D tumor segmentation using the composite metrics, we first quantify the relation between these two measures. Because the radiologist's rating is based on ranking of the quality of the segmentation, the relation can be described using the correlation of the order of the same set of segmentation defined by radiologist's rating and the composite metric.

One method to quantitatively compute the strength of such correlation is to use Spearman rank correlation coefficient. See [12] for details on Spearman rank correlation coefficient.

To avoid negative value in the computed rank correlation, all objective metrics used in this study except volume overlap are linearly mapped into the interval $[0,1]$, in which higher value is associated with better segmentation quality. From (5), the composite measure computed using normalized objective metrics on all lesions can be expressed as

$$
\overline{\mathbf{u}}=\overline{\mathbf{M}} \mathbf{c}
$$

where $\overline{\mathbf{u}}$ is a $Q \times 1$ vector of the measurements from the composite metric, $\overline{\mathbf{M}}$ is a $Q \times n$ matrix of normalized objective measurements.

Let $\mathbf{w}$, a $Q \times 1$ vector, denote the radiologist's five-level ratings for segmentation of all the $Q$ lesions. The Spearman rank correlation coefficient between the subjective and composite measures is computed as

$$
r_{s}(\mathbf{w}, \overline{\mathbf{u}})=1-\frac{6 \sum_{i=1}^{Q}\left[\mathcal{R}_{i}(\mathbf{w})-\mathcal{R}_{i}(\overline{\mathbf{u}})\right]^{2}}{Q\left(Q^{2}-1\right)},
$$

where $r_{s}$ is the Spearman rank correlation coefficient, $\mathcal{R}_{i}(\cdot)$ denotes the rank of the $i$-th element in the vector.

For tied ranks in either subjective or composite group, ranks are computed using the mean value of the ranks those segmentations would have had, if they had been distinguishable [12].

We can make the two sets of rankings as similar as possible by maximizing the Spearman rank correlation coefficient in (7). For a fixed set of objective metrics, i.e., $n$ and $m_{i}, i=1, \ldots, n$ are fixed, the optimal weighing parameters are determined by

$$
\mathbf{c}^{*}=\underset{\mathbf{c}}{\arg \max }\left\{r_{s}(\mathbf{w}, \overline{\mathbf{u}})\right\},
$$

Using the results from (5) and (77), the optimization problem in (8) is equivalent to

$$
\mathbf{c}^{*}=\underset{\mathbf{c}}{\arg \min }\left\{\sum_{i=1}^{Q}\left[\mathcal{R}_{i}(\mathbf{w})-\mathcal{R}_{i}(\overline{\mathbf{M}} \mathbf{c})\right]^{2}\right\} \text {. }
$$


We use "lsqnonlin", a non-linear least square optimization function in MATLAB2, to compute the optimal weighing parameters in (9). Once the weighing parameters are determined, the value of the composite metric for segmentation of all lesions can be obtained from the objective measurements using Eq. (6).

\subsection{Evaluation of Segmentation}

The composite metric computed from all training data is used to determine five cluster centers for segmentation evaluation. Each cluster center is computed using the mean value of the composite metric from lesions in each of the five categories defined by the radiologist. All five centers are sorted in ascending order and assigned labels of 1 to 5 to simulate the radiologist's five-level scale.

To rate segmentation of a testing lesion, the value of the composite metric for this lesion is first computed using the method described in previous section. A list of five possible scores is generated in such a way that the label of the nearest cluster center is on the top, and the label of the farthest center at the bottom. Note that the top two scores in the list are always adjacent scores, e.g., 1 and 2, 4 and 3, due to the monotonically increased composite metric value for the five cluster centers. This property is desirable in simulating radiologist's rating because it is common in clinical practice for a radiologist to adjust the rating by one level, as the result of fuzzily defined adjacent classes in the 5-point scale.

\section{$3 \quad$ Results}

\subsection{Accuracy of Segmentation Evaluation}

We tested our segmentation evaluation method using a set of 93 tumors from patients with a variety of cancer, including liver, lymphoma, lung, and so on. All the image datasets were acquired on a 64-slice Siemens SOMATOM Sensation CT scanner. The results of the radiologist's subjective evaluation for all 93 lesions using the five-level scale are briefly described as follows. The number of lesions with rating from 1 to 5 are $7,10,19,39$ and 18 respectively.

We evaluated our technique using leave-one-out cross validation [13. The performance was measured using average correct rank and frequency of correct score in the top ranks of the rating list [14]. The average correct rank is computed as the rank of the correct score, i.e., the radiologist's rating, in the generated rating list averaged over all 93 trials. Smaller value of average correct rank indicates better performance of evaluation. For comparison purpose, Spearman rank correlation coefficient between radiologist's rating and quantitative measures averaged over all 93 trials was computed as well.

To select the best set of objective metrics for the composite metric, the leaveone-out procedure was repeated on all combinations of the candidate objective metrics. This is feasible because of the relatively small number of objective metrics used in this study. Rating segmentation using single objective metric was

\footnotetext{
${ }^{2}$ MATLAB is a product of The Mathworks, Natick, MA.
} 
implemented in a way similar to that for composite metric described in Section 2.3

The segmentation evaluation results are shown in Table 1 and Fig. 2, For the sake of brevity, Fig. 2 only shows results from three combinations with smallest average rank of correct scores. These three combinations also showed the highest percentage of correct score in the top ranks of rating list among all combinations using either single or multiple objective metrics. Compared to other combinations, the composite metric computed using all three objective metrics showed the best value for each performance measure. The average correct rank is 1.44 , and the average Spearman correlation coefficient is 0.83 . The percentage of correct rating in the first place of the list is $66.7 \%$, and increases to $90.8 \%$ for top two choices. This result suggests that there is $\sim 90 \%$ of chance that one of the two ratings in the top two positions in the list will match radiologist's score.

Table 1. Rank of correct label in the rating list and Spearman rank correlation coefficient $\left(r_{s}\right)$ between radiologist's qualitative rating and quantitative measures for all combinations of objective metrics averaged over all 93 leave-one-out trials. A $p$ value less than 0.05 is considered significant. $u$ denotes composite measure from objective metrics $m_{1}, m_{2}$ and $m_{3}$.

\begin{tabular}{cccc}
\hline Metric & Average rank & $r_{s}$ & $p$ \\
\hline$m_{1}$ & 1.70 & 0.80 & $<0.0001$ \\
$m_{2}$ & 1.60 & 0.75 & $<0.0001$ \\
$m_{3}$ & 1.98 & $0.70<0.0001$ \\
$u_{m_{1}, m_{2}}$ & 1.48 & $0.83<0.0001$ \\
$u_{m_{1}, m_{3}}$ & 1.62 & $0.80<0.0001$ \\
$u_{m_{2}, m_{3}}$ & 1.55 & 0.77 & $<0.0001$ \\
$u_{m_{1}, m_{2}, m_{3}}$ & 1.44 & $0.83<0.0001$ \\
\hline
\end{tabular}

The performance of composite metric from objective metrics $m_{1}, m_{2}$ and $m_{3}$ was slightly better than that from $m_{1}$ and $m_{2}$. This is probably because the RMS surface distance $\left(m_{3}\right)$ and volume overlap $\left(m_{1}\right)$ both reflect the relative position of two segmentations, although $m_{3}$ may be more sensitive than $m_{1}$. Using more similar metrics in the composite metric provides only limited help for improving the performance.

From the experimental results above, we chose all the three objective metrics as the optimal combination, and used this combination for the remaining experiments. The composite metric determined from all the 93 lesions is given by

$$
u_{m_{1}, m_{2}, m_{3}}=0.39 m_{1}+0.15 m_{2}+0.42 m_{3} .
$$

\subsection{Parameter Sensitivity}

We studied the variation of the optimal weighing parameters of the composite metric and the five cluster centers for rating segmentation in the leave-one-out cross validation. The variation is measured by coefficient of variation, which is defined as ratio of standard variation to the mean of data. Table 2 shows the 


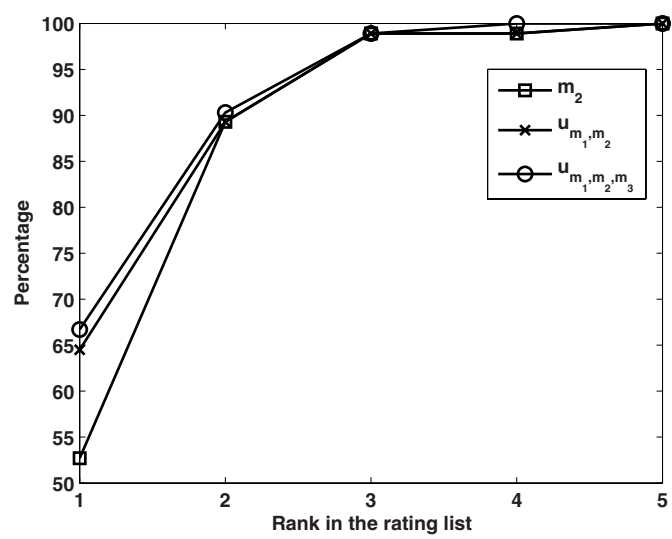

Fig. 2. Frequency of the correct score in the top ranks of the rating list for three representative combinations of objective metrics

Table 2. Coefficient of variation of weighing parameters in the composite metric and five cluster centers for rating segmentation in the 93 leave-one-out trials

\begin{tabular}{|ccc||ccccc|}
\hline \multicolumn{3}{|c||}{ Weight } & \multicolumn{5}{c|}{ Cluster Center } \\
\hline$c_{1}$ & $c_{2}$ & $c_{3}$ & $c t r_{1}$ & $c t r_{2}$ & $c t r_{3}$ & $c t r_{4}$ & $c t r_{5}$ \\
\hline $7.6 \%$ & $8.7 \%$ & $17.4 \%$ & $8.8 \%$ & $9.1 \%$ & $8.9 \%$ & $8.8 \%$ & $8.8 \%$ \\
\hline
\end{tabular}

coefficient of variation for the weighing parameters and cluster centers. Results from 4 of the 93 trials showed significantly different values than others, and were removed as outliers. The weighing parameter for RMS surface distance showed larger variation than those for volume overlap and absolute value of normalized volume difference. This is mainly because RMS surface distance is more sensitive to lesion size. The value of all the five cluster centers showed relatively small change.

\section{Discussion and Conclusion}

We presented a method for evaluating 3D tumor segmentation using a new composite metric. The composite metric is defined as a linear combination of a set of objective metrics. The segmentation is rated using the composite metric on a fivepoint scale to simulate the radiologist's criterion routinely used in clinical setting.

Experimental results on 93 lesions demonstrated that the new composite metric showed better performance in rating segmentation than each individual objective metric. Our method is also relatively robust to the selection of training data. These results suggest that our method can provide a more adequate and reproducible evaluation of the quality of tumor segmentation.

The combined objective metrics for segmentation evaluation in 67896] were determined empirically. In comparison, the weighing parameters of the new 
composite metric were computed by quantitatively correlating the objective and subjective measures. Consequently, our method can be applied to evaluate different segmentation algorithms and segmentation results on images from other imaging modalities, such as MRI.

Results on 93 lesions showed that segmentation rating from the new composite metric compared well with radiologist's score using top two selection in the rating list. This result suggests that our method can facilitate segmentation evaluation on large scale clinical study by predicting radiologist's five-level rating, especially when such scores are not available for all the data.

Future work includes deriving a composite metric that can simulate subjective evaluation from multiple radiologists, and investigating the reproducibility of radiologist's rating and the optimal sample size in the training stage.

\section{References}

1. Therasse, P., et al.: New guideline to evaluate the response to treatment in solid tumors. Journal of National Cancer Institute 92(3), 205-216 (2000)

2. World Health Organization: WHO Handbook for Reporting Results of Cancer Treatment. World Health Organization (1979)

3. Van Hoe, L., et al.: Size quantification of liver metastases in patients undergoing cancer treatment: reproducibility of one-, two-, and three-dimensional measurements determined with spiral CT. Radiology 202(3), 671-675 (1997)

4. Zhang, Y.J.: A survey on evaluation methods for image segmentation. Pattern Recognition 29(8), 1335-1346 (1996)

5. Armato, S.G., Griger, M.L., MacMahon, H.: Automated lung segmentation in digitized posteroanterior chest radiographs. Academic Radiology 5(4), 245-255 (1998)

6. Correia, P.L., Pereira, F.: Objective evaluation of video segmentation quality. IEEE Transactions on Image Processing 12(2), 186-200 (2003)

7. Villegas, P., Marichal, X.: Perceptually-weighted evaluation criteria for segmentation masks in video sequences. IEEE Transactions on Image Processing 13(8), 1092 $1103(2004)$

8. Cavallaro, A., Gelasca, E.D., Ebrahimi, T.: Objective evaluation of segmentation quality. In: Proceedings of IEEE ICIP, pp. 301-304. IEEE Computer Society Press, Los Alamitos (2002)

9. Gelasca, E.D., et al.: A framework for evaluating video object segmentation algorithms. In: Proceedings of IEEE CVPRW, pp. 191-198. IEEE Computer Society Press, Los Alamitos (2006)

10. Grady, L.: Random walks for image segmentation. IEEE Transactions on Pattern Analysis and Machine Intelligence 28(11), 1768-1783 (2006)

11. Delgorge, C., et al.: Towards a new tool for the evaluation of the quality of ultrasound compressed images. IEEE Transactions on Medical Imaging 25(11), 1502 1509 (2006)

12. Sheskin, D.I.: Handbook of Parametric and Nonparametric Statistical Procedures. CRC Press (1997)

13. Duda, R.O., Hart, P.E., Stork, D.G.: Pattern Classification, 2nd edn. John Wiley and Sons, Chichester (2001)

14. Yoon, S., et al.: Automatic skin pixel selection and skin color classification. In: Proceedings of IEEE ICIP, pp. 941-944. IEEE Computer Society Press, Los Alamitos (2006) 